\title{
THE WELL-COVERED DIMENSION OF PRODUCTS OF GRAPHS
}

\author{
Isaac Birnbaum $^{1}$, Megan Kuneli ${ }^{1}$ \\ Robyn McDonald ${ }^{2}$, Katherine Urabe ${ }^{1}$ \\ AND \\ OSCAR VEGA ${ }^{1}$ \\ 1 Department of Mathematics \\ California State University, Fresno \\ Fresno, CA, USA \\ 2 California State University, Stanislaus \\ Turlock, CA, USA \\ e-mail: isaacb1@mail.fresnostate.edu \\ mrkunelius@mail.fresnostate.edu \\ rmcdonald@csustan.edu \\ kturabe@mail.fresnostate.edu \\ ovega@csufresno.edu
}

\begin{abstract}
We discuss how to find the well-covered dimension of a graph that is the Cartesian product of paths, cycles, complete graphs, and other simple graphs. Also, a bound for the well-covered dimension of $K_{n} \times G$ is found, provided that $G$ has a largest greedy independent decomposition of length $c<n$.

Formulae to find the well-covered dimension of graphs obtained by vertex blowups on a known graph, and to the lexicographic product of two known graphs are also given.
\end{abstract}

Keywords: well-covered dimension, maximal independent sets.

2010 Mathematics Subject Classification: 05C50, 15 A03.

\section{REFERENCES}

[1] J.I. Brown and R.J. Nowakowski, Well-covered vector spaces of graphs, SIAM J. Discrete Math. 19 (2005) 952-965.

doi:10.1137/S0895480101393039 
[2] Y. Caro, M.N. Ellingham and J.E. Ramey, Local structure when all maximal independent sets have equal weight, SIAM J. Discrete Math. 11 (1998) 644-654. doi:10.1137/S0895480196300479

[3] Y. Caro and R. Yuster, The uniformity space of hypergraphs and its applications, Discrete Math. 202 (1999) 1-19.

doi:10.1016/S0012-365X(98)00344-6

[4] A. Ovetsky, On the well-coveredness of Cartesian products of graphs, Discrete Math. 309 (2009) 238-246.

doi:10.1016/j.disc.2007.12.083

[5] M.D. Plummer, Some covering concepts in graphs, J. Combin. Theory 8 (1970) 9198 .

doi:10.1016/S0021-9800(70)80011-4

[6] D.B. West, Introduction to Graph Theory, Second Edition (Prentice Hall, Upper Saddle River, 2001).

Received 13 December 2012

Revised 19 November 2013

Accepted 19 November 2013 